The Russian option: Finite horizon

Peskir, Goran

2005

MIMS EPrint: 2007.37

Manchester Institute for Mathematical Sciences

School of Mathematics

The University of Manchester

\footnotetext{
Reports available from: http://eprints.maths.manchester.ac.uk/

And by contacting: The MIMS Secretary

School of Mathematics

The University of Manchester

Manchester, M13 9PL, UK
}

ISSN 1749-9097 


\title{
The Russian option: Finite horizon
}

\author{
Goran Peskir ${ }^{\star}$ \\ Department of Mathematical Sciences, University of Aarhus, Ny Munkegade, 8000 Aarhus, Denmark \\ (e-mail: goran@imf.au.dk)
}

\begin{abstract}
We show that the optimal stopping boundary for the Russian option with finite horizon can be characterized as the unique solution of a nonlinear integral equation arising from the early exercise premium representation (an explicit formula for the arbitrage-free price in terms of the optimal stopping boundary having a clear economic interpretation). The results obtained stand in a complete parallel with the best known results on the American put option with finite horizon. The key argument in the proof relies upon a local time-space formula.
\end{abstract}

Key words: Russian option, finite horizon, arbitrage-free price, optimal stopping, smooth-fit, geometric Brownian motion, free-boundary problem, nonlinear integral equation, local time-space calculus, curved boundary

\section{JEL Classification: G13}

Mathematics Subject Classification (2000): 91B28, 35R35, 45G10, 60G40, $60 \mathrm{~J} 60$

\section{Introduction}

According to the financial theory (see e.g. [14] or [6]) the arbitrage-free price of the Russian option is given by (2.1) below where $M$ denotes the maximum of the stock price $S$. This option is characterized by 'reduced regret' because its owner

\footnotetext{
${ }^{\star}$ Centre for Analytical Finance (funded by the Danish Social Science Research Council) and Network in Mathematical Physics and Stochastics (funded by the Danish National Research Foundation).

The first draft of the present paper has been completed in September 2002. I am indebted to Albert Shiryaev for useful comments.
} 
is paid the maximum stock price up to the time of exercise and hence feels less remorse for not having exercised the option earlier.

In the case of infinite horizon $T$, and when $M_{\tau}$ in (2.1) is replaced by $e^{-\lambda \tau} M_{\tau}$, the problem was solved by Shepp and Shiryaev. The original derivation [11] was two-dimensional (see [9] for a general principle in this context) and the subsequent derivation [12] reduced the problem to one dimension using a change-of-measure theorem. The latter methodology will also be adopted in the present article.

Apart from the fact that practitioners find finite horizons more desirable, the infinite horizon formulation requires the discounting rate $\lambda>0$ to be present, since otherwise the option price would be infinite. Clearly, such a discounting rate is not needed when the horizon $T$ is finite, so that the most attractive feature of the option - no regret - remains fully preserved.

The work of Shepp and Shiryaev [12] showed that the Russian option problem becomes one-dimensional after the change-of-measure theorem is applied (see (2.4)-(2.7) below) thus setting the mathematical problem on an equal footing with the American option problem (put or call) with finite horizon. The latter problem, on the other hand, has been studied since the 1960's, and for more details and references including the latest definite results we refer to [10]. The main aim of the present article is to extend these results to the Russian option with finite horizon.

In Sect. 2 we formulate the Russian option problem with finite horizon and recall some known facts needed later. In Sect. 3 we present the main result and proof. The key argument in the proof of uniqueness relies upon a local time-space formula (see [10]). To obtain a more complete understanding of the results given here we refer to [10] for mathematical complements and to [1] for financial interpretations. Both carry over to the present case with no major change.

\section{Formulation of the problem}

The arbitrage-free price of the Russian option with finite horizon is given by:

$$
V=\sup _{0 \leq \tau \leq T} E\left(e^{-r \tau} M_{\tau}\right)
$$

where $\tau$ is a stopping time of the geometric Brownian motion $S=\left(S_{t}\right)_{0 \leq t \leq T}$ solving:

$$
d S_{t}=r S_{t} d t+\sigma S_{t} d B_{t} \quad\left(S_{0}=s\right)
$$

and $M=\left(M_{t}\right)_{0 \leq t \leq T}$ is the maximum process given by:

$$
M_{t}=\left(\max _{0 \leq u \leq t} S_{u}\right) \vee m
$$

where $m \geq s>0$ are given and fixed. [Throughout $B=\left(B_{t}\right)_{t \geq 0}$ denotes a standard Brownian motion started at zero.] We recall that $T>0$ is the expiration date (maturity), $r>0$ is the interest rate, and $\sigma>0$ is the volatility coefficient. 
For the purpose of comparison with the infinite-horizon results [11] we will also introduce a discounting rate $\lambda \geq 0$ so that $M_{\tau}$ in (2.1) is to be replaced by $e^{-\lambda \tau} M_{\tau}$. By the change-of-measure theorem it then follows that:

$$
V=\sup _{0 \leq \tau \leq T} \widetilde{E}\left(e^{-\lambda \tau} X_{\tau}\right)
$$

where following the key fact of [12] we set:

$$
X_{t}=\frac{M_{t}}{S_{t}}
$$

and $\widetilde{P}$ is defined by $d \widetilde{P}=\exp \left(\sigma B_{T}-\left(\sigma^{2} / 2\right) T\right) d P$ so that $\widetilde{B}_{t}=B_{t}-\sigma t$ is a standard Brownian motion under $\widetilde{P}$ for $0 \leq t \leq T$. By Itô's formula one finds that $X$ solves:

$$
d X_{t}=-r X_{t} d t+\sigma X_{t} d \widehat{B}_{t}+d R_{t} \quad\left(X_{0}=x\right)
$$

under $\widetilde{P}$ where $\widehat{B}=-\widetilde{B}$ is a standard Brownian motion, and we set:

$$
R_{t}=\int_{0}^{t} I\left(X_{s}=1\right) \frac{d M_{s}}{S_{s}}
$$

for $0 \leq t \leq T$. It follows that $X$ is a diffusion in $[1, \infty\rangle$ having 1 as a boundary point of instantaneous reflection. The infinitesimal generator of $X$ is therefore given by:

$$
\begin{aligned}
\mathbb{L}_{X} & =-r x \frac{\partial}{\partial x}+\frac{\sigma^{2}}{2} x^{2} \frac{\partial^{2}}{\partial x^{2}} \text { in }\langle 1, \infty\rangle \\
\frac{\partial}{\partial x} & =0 \text { at } 1+.
\end{aligned}
$$

[The latter means that the infinitesimal generator of $X$ is acting on a space of $C^{2}$ functions $f$ defined on $[1, \infty\rangle$ such that $f^{\prime}(1+)=0$.] For more details on the derivation (2.4)-(2.7) see [12].

For further reference recall that the strong solution of (2.2) is given by:

$$
S_{t}=s \exp \left(\sigma B_{t}+\left(r-\sigma^{2} / 2\right) t\right)=s \exp \left(\sigma \widetilde{B}_{t}+\left(r+\sigma^{2} / 2\right) t\right)
$$

for $0 \leq t \leq T$ under $P$ and $\widetilde{P}$ respectively. When dealing with the process $X$ on its own, however, note that there is no restriction to assume that $s=1$ and $m=x$ with $x \geq 1$.

Summarizing the preceding facts we see that the Russian option problem with finite horizon reduces to solve the following optimal stopping problem (extended in accordance with a well-known argument from general theory):

$$
V(t, x)=\sup _{0 \leq \tau \leq T-t} \widetilde{E}_{t, x}\left(e^{-\lambda \tau} X_{t+\tau}\right)
$$

where $\tau$ is a stopping time of the diffusion process $X$ satisfying (2.5)-(2.8) above and $X_{t}=x$ under $\widetilde{P}_{t, x}$ with $(t, x) \in[0, T] \times[1, \infty\rangle$ given and fixed. 
Standard Markovian arguments (cf. [3]) indicate that $V$ from (2.10) solves the following free-boundary problem of parabolic type:

$$
\begin{aligned}
& V_{t}+\mathbb{L}_{X} V=\lambda V \text { in } C \\
& V(t, x)=x \text { for } x=b(t) \text { or } t=T \\
& V_{x}(t, x)=1 \text { for } x=b(t) \quad \text { (smooth-fit) } \\
& V_{x}(t, 1+)=0 \quad \text { (normal reflection) } \\
& V(t, x)>x \text { in } C \\
& V(t, x)=x \text { in } D
\end{aligned}
$$

where the continuation set $C$ and the stopping set $S=\bar{D}$ are defined by:

$$
\begin{aligned}
& C=\{(t, x) \in[0, T\rangle \times[1, \infty\rangle \mid x<b(t)\} \\
& D=\{(t, x) \in[0, T\rangle \times[1, \infty\rangle \mid x>b(t)\}
\end{aligned}
$$

and $b:[0, T] \rightarrow \mathbb{R}$ is the (unknown) optimal stopping boundary, i.e. the stopping time:

$$
\tau_{b}=\inf \left\{0 \leq s \leq T-t \mid X_{t+s} \geq b(t+s)\right\}
$$

is optimal in (2.10) (i.e. the supremum is attained at this stopping time).

It will follow from the result of Theorem 3.1 below that the free-boundary problem (2.11)-(2.16) characterizes the value function $V$ and the optimal stopping boundary $b$ in a unique manner. Our main aim, however, is to follow the train of thought initiated by Kolodner [8] where $V$ is firstly expressed in terms of $b$, and $b$ itself is shown to satisfy a nonlinear integral equation. A particularly simple approach for achieving this goal in the case of the American put option has been suggested in $[7,5,1]$ and we will take it up in the present paper as well. We will moreover see (as in [10]) that the nonlinear equation derived for $b$ cannot have other solutions.

\section{The result and proof}

In this section we adopt the setting and notation of the Russian option problem from the previous section. Below we will make use of the following functions:

$$
\begin{aligned}
F(t, x) & =\widetilde{E}_{0, x}\left(X_{t}\right)=\int_{1}^{\infty} \int_{0}^{m}\left(\frac{m \vee x}{s}\right) f(t, s, m) d s d m \\
G(t, x, y) & =\widetilde{E}_{0, x}\left(X_{t} I\left(X_{t} \geq y\right)\right) \\
& =\int_{1}^{\infty} \int_{0}^{m}\left(\frac{m \vee x}{s}\right) I\left(\left(\frac{m \vee x}{s}\right) \geq y\right) f(t, s, m) d s d m
\end{aligned}
$$

for $t>0$ and $x, y \geq 1$, where $(s, m) \mapsto f(t, s, m)$ is the probability density function of $\left(S_{t}, M_{t}\right)$ under $\widetilde{P}$ with $S_{0}=M_{0}=1$ given by (see e.g. [6] p. 368):

$$
f(t, s, m)=\frac{2}{\sigma^{3} \sqrt{2 \pi t^{3}}} \frac{\log \left(m^{2} / s\right)}{s m} \exp \left(-\frac{\log ^{2}\left(m^{2} / s\right)}{2 \sigma^{2} t}+\frac{\beta}{\sigma} \log (s)-\frac{\beta^{2}}{2} t\right)
$$


for $0<s \leq m$ and $m \geq 1$ with $\beta=r / \sigma+\sigma / 2$, and is equal to 0 otherwise.

The main result of the paper may now be stated as follows.

Theorem 3.1 The optimal stopping boundary in the Russian option problem (2.10) can be characterized as the unique continuous decreasing solution $b:[0, T] \rightarrow \mathbb{R}$ of the nonlinear integral equation:

$$
b(t)=e^{-\lambda(T-t)} F(T-t, b(t))+(r+\lambda) \int_{0}^{T-t} e^{-\lambda u} G(u, b(t), b(t+u)) d u
$$

satisfying $b(t)>1$ for all $0<t<T$. [The solution $b$ satisfies $b(T-)=1$ and the stopping time $\tau_{b}$ from (2.19) is optimal in (2.10) (see Fig. 1 below).]

The arbitrage-free price of the Russian option (2.10) admits the following 'early exercise premium' representation:

$$
V(t, x)=e^{-\lambda(T-t)} F(T-t, x)+(r+\lambda) \int_{0}^{T-t} e^{-\lambda u} G(u, x, b(t+u)) d u
$$

for all $(t, x) \in[0, T] \times[1, \infty\rangle$. [Further properties of $V$ and $b$ are exhibited in the proof below.]

Proof The proof will be carried out in several steps. We begin by stating some general remarks which will be freely used below without further mentioning.

It is easily seen that $E\left(\max _{0 \leq t \leq T} X_{t}\right)<\infty$ so that $V(t, x)<\infty$ for all $(t, x) \in[0, T] \times[1, \infty)$. Recall that it is no restriction to assume that $s=1$ and $m=x$ so that $X_{t}=\left(M_{t} \vee x\right) / S_{t}$ with $S_{0}=M_{0}=1$. We will write $X_{t}^{x}$ instead of $X_{t}$ to indicate the dependence on $x$ when needed. Since $M_{t} \vee x=\left(x-M_{t}\right)^{+}+M_{t}$ we see that $V$ admits the following representation:

$$
V(t, x)=\sup _{0 \leq \tau \leq T-t} \widetilde{E}\left(e^{-\lambda \tau} \frac{\left(x-M_{\tau}\right)^{+}+M_{\tau}}{S_{\tau}}\right)
$$

for $(t, x) \in[0, T] \times[1, \infty\rangle$. It follows immediately from (3.6) that:

$$
x \mapsto V(t, x) \text { is increasing and convex on }[1, \infty\rangle
$$

for each $t \geq 0$ fixed. It is also obvious from (3.6) that $t \mapsto V(t, x)$ is decreasing on $[0, T]$ with $V(T, x)=x$ for each $x \geq 1$ fixed.

1 . We show that $V:[0, T] \times[1, \infty\rangle \rightarrow \mathbb{R}$ is continuous. For this, using $\sup (f)-$ $\sup (g) \leq \sup (f-g)$ and $(y-z)^{+}-(x-z)^{+} \leq(y-x)^{+}$for $x, y, z \in \mathbb{R}$, we get:

$$
V(t, y)-V(t, x) \leq(y-x) \sup _{0 \leq \tau \leq T-t} \widetilde{E}\left(e^{-\lambda \tau}\left(1 / S_{\tau}\right)\right) \leq y-x
$$

for $1 \leq x<y$ and all $t \geq 0$, where in the second inequality we used (2.9) to deduce that $1 / S_{t}=\exp \left(\sigma \hat{B}_{t}-\left(r+\sigma^{2} / 2\right) t\right) \leq \exp \left(\sigma \hat{B}_{t}-\left(\sigma^{2} / 2\right) t\right)$ and the latter is a 
martingale under $\widetilde{P}$. From (3.8) with (3.7) we see that $x \mapsto V(t, x)$ is continuous uniformly over $t \in[0, T]$. Thus to prove that $V$ is continuous on $[0, T] \times[1, \infty\rangle$ it is enough to show that $t \mapsto V(t, x)$ is continuous on $[0, T]$ for each $x \geq 1$ given and fixed. For this, take any $t_{1}<t_{2}$ in $[0, T]$ and $\varepsilon>0$, and let $\tau_{1}^{\varepsilon}$ be a stopping time such that $\widetilde{E}\left(e^{-\lambda \tau_{1}^{\varepsilon}} X_{t_{1}+\tau_{1}^{\varepsilon}}^{x}\right) \geq V\left(t_{1}, x\right)-\varepsilon$. Setting $\tau_{2}^{\varepsilon}=\tau_{1}^{\varepsilon} \wedge\left(T-t_{2}\right)$ we see that $V\left(t_{2}, x\right) \geq \widetilde{E}\left(e^{-\lambda \tau_{2}^{\varepsilon}} X_{t_{2}+\tau_{2}^{\varepsilon}}^{x}\right)$. Hence we get:

$$
0 \leq V\left(t_{1}, x\right)-V\left(t_{2}, x\right) \leq \widetilde{E}\left(e^{-\lambda \tau_{1}^{\varepsilon}} X_{t_{1}+\tau_{1}^{\varepsilon}}^{x}-e^{-\lambda \tau_{2}^{\varepsilon}} X_{t_{2}+\tau_{2}^{\varepsilon}}^{x}\right)+\varepsilon
$$

Letting first $t_{2}-t_{1} \rightarrow 0$ using $\tau_{1}^{\varepsilon}-\tau_{2}^{\varepsilon} \rightarrow 0$ and then $\varepsilon \downarrow 0$ we see that $V\left(t_{1}, x\right)-V\left(t_{2}, x\right) \rightarrow 0$ by dominated convergence. This shows that $t \mapsto V(t, x)$ is continuous on $[0, T]$, and the proof of the initial claim is complete.

Denote $G(x)=x$ for $x \geq 1$ and introduce the continuation set $C=\{(t, x) \in$ $[0, T\rangle \times[1, \infty\rangle \mid V(t, x)>G(x)\}$ and the stopping set $S=\{(t, x) \in[0, T\rangle \times$ $[1, \infty\rangle \mid V(t, x)=G(x)\}$. Since $V$ and $G$ are continuous, we see that $C$ is open and $S$ is closed in $[0, T\rangle \times[1, \infty\rangle$. Standard arguments based on the strong Markov property (cf. [13]) show that the first hitting time $\tau_{S}=\inf \{0 \leq s \leq$ $\left.T-t \mid\left(t+s, X_{t+s}\right) \in S\right\}$ is optimal in (2.10).

2. We show that the continuation set $C$ just defined is given by (2.17) for some decreasing function $b:[0, T\rangle \rightarrow\langle 1, \infty\rangle$. It follows in particular that the stopping set $S$ coincides with the closure $\bar{D}$ in $[0, T\rangle \times[1, \infty\rangle$ of the set $D$ in $(2.18)$ as claimed. To verify the initial claim, note that by Itô's formula and (2.6) we have:

$$
e^{-\lambda s} X_{t+s}=X_{t}-(r+\lambda) \int_{0}^{s} e^{-\lambda u} X_{t+u} d u+\int_{0}^{s} e^{-\lambda u} \frac{d M_{t+u}}{S_{t+u}}+N_{s}
$$

where $N_{s}=\sigma \int_{0}^{s} e^{-\lambda u} X_{t+u} d \widehat{B}_{t+u}$ is a martingale for $0 \leq s \leq T-t$. Let $t \in[0, T]$ and $x>y \geq 1$ be given and fixed. We will first show that $(t, x) \in C$ implies that $(t, y) \in C$. For this, let $\tau_{*}=\tau_{*}(t, x)$ denote the optimal stopping time for $V(t, x)$. Taking the expectation in (3.10) stopped at $\tau_{*}$, first under $\widetilde{P}_{t, y}$ and then under $\widetilde{P}_{t, x}$, and using the optional sampling theorem to get rid of the martingale part, we find:

$$
\begin{aligned}
V(t, y)-y & \geq \widetilde{E}_{t, y}\left(e^{-\lambda \tau_{*}} X_{t+\tau_{*}}\right)-y \\
& =-(r+\lambda) \widetilde{E}_{t, y}\left(\int_{0}^{\tau_{*}} e^{-\lambda u} X_{t+u} d u\right)+\widetilde{E}_{t, y}\left(\int_{0}^{\tau_{*}} e^{-\lambda u} \frac{d M_{t+u}}{S_{t+u}}\right) \\
& \geq-(r+\lambda) \widetilde{E}_{t, x}\left(\int_{0}^{\tau_{*}} e^{-\lambda u} X_{t+u} d u\right)+\widetilde{E}_{t, x}\left(\int_{0}^{\tau_{*}} e^{-\lambda u} \frac{d M_{t+u}}{S_{t+u}}\right) \\
& =\widetilde{E}_{t, x}\left(e^{-\lambda \tau_{*}} X_{t+\tau_{*}}\right)-x=V(t, x)-x>0
\end{aligned}
$$

proving the claim. To explain the second inequality in (3.11) note that the process $X$ under $\widetilde{P}_{t, z}$ can be realized as the process $X^{t, z}$ under $P$ where we set $X_{t+u}^{t, z}=$ $\left(S_{u}^{*} \vee z\right) / S_{u}$ with $S_{u}^{*}=\max _{0 \leq v \leq u} S_{v}$. Then note that $X_{t+u}^{t, y} \leq X_{t+u}^{t, x}$ and $d\left(S_{u}^{*} \vee\right.$ $y) \geq d\left(S_{u}^{*} \vee x\right)$ whenever $y \leq x$, and thus each of the two terms on the left-hand 
side of the inequality is larger than the corresponding term on the right-hand side, implying the inequality. The fact just proved establishes the existence of a function $b:[0, T] \rightarrow[1, \infty]$ such that the continuation set $C$ is given by (2.17) above.

Let us show that $b$ is decreasing. For this, with $x \geq 1$ and $t_{1}<t_{2}$ in $[0, T]$ given and fixed, it is enough to show that $\left(t_{2}, x\right) \in C$ implies that $\left(t_{1}, x\right) \in C$. To verify this implication, recall that $t \mapsto V(t, x)$ is decreasing on $[0, T]$, so that we have:

$$
V\left(t_{1}, x\right) \geq V\left(t_{2}, x\right)>x
$$

proving the claim.

Let us show that $b$ does not take the value $\infty$. For this, assume that there exists $t_{0} \in\langle 0, T]$ such that $b(t)=\infty$ for all $0 \leq t \leq t_{0}$. It implies that $(0, x) \in C$ for any $x \geq 1$ given and fixed, so that if $\tau_{*}=\tau_{*}(0, x)$ denote the optimal stopping time for $V(0, x)$, we have $V(0, x)>x$ which by (3.10) is equivalent to:

$$
\widetilde{E}_{0, x}\left(\int_{0}^{\tau_{*}} e^{-\lambda u} \frac{d M_{u}}{S_{u}}\right)>(r+\lambda) \widetilde{E}_{0, x}\left(\int_{0}^{\tau_{*}} e^{-\lambda u} X_{u} d u\right) .
$$

Recalling that $M_{u}=S_{u}^{*} \vee x$ we see that:

$$
\begin{aligned}
\widetilde{E}_{0, x}\left(\int_{0}^{\tau_{*}} e^{-\lambda u} \frac{d M_{u}}{S_{u}}\right) & \leq \widetilde{E}\left(\left(\max _{0 \leq u \leq T}\left(1 / S_{u}\right)\right)\left(\left(S_{T}^{*} \vee x\right)-x\right)\right) \\
& \leq \widetilde{E}\left(\left(\max _{0 \leq u \leq T}\left(1 / S_{u}\right)\right) S_{T}^{*} I\left(S_{T}^{*}>x\right)\right) \rightarrow 0
\end{aligned}
$$

as $x \rightarrow \infty$. Recalling that $X_{u}=\left(S_{u}^{*} \vee x\right) / S_{u}$ and noting that $\tau_{*}>t_{0}$ we see that:

$$
\widetilde{E}_{0, x}\left(\int_{0}^{\tau_{*}} e^{-\lambda u} X_{u} d u\right) \geq e^{-\lambda t_{0}} x \widetilde{E}\left(\int_{0}^{t_{0}} \frac{d u}{S_{u}}\right) \rightarrow \infty
$$

as $x \rightarrow \infty$. From (3.14) and (3.15) we see that the strict inequality in (3.13) is violated if $x$ is taken large enough, thus proving that $b$ does not take the value $\infty$ on $\langle 0, T]$. To disprove the case $b(0+)=\infty$, i.e. $t_{0}=0$ above, we may note that the gain function $G(x)=x$ in (2.10) is independent of time, so that $b(0+)=\infty$ would also imply that $b(t)=\infty$ for all $0 \leq t \leq \delta$ in the problem (2.10) with the horizon $T+\delta$ instead of $T$ where $\delta>0$. Applying the same argument as above to the $T+\delta$ problem (2.10) we again arrive to a contradiction. We thus may conclude that $b(0+)<\infty$ as claimed. Yet another quick argument for $b$ to be finite in the case $\lambda>0$ can be given by noting that $b(t)<\alpha$ for all $t \in[0, T]$ where $\alpha \in\langle 1, \infty\rangle$ is the optimal stopping point in the infinite horizon problem given explicitly in (2.3) of [11]. Clearly $b(t) \uparrow \alpha$ as $T \rightarrow \infty$ for each $t \geq 0$, where we set $\alpha=\infty$ in the case $\lambda=0$.

Let us show that $b$ cannot take the value 1 on $[0, T\rangle$. This fact is equivalent to the fact that the process $\left(S_{t}, M_{t}\right)$ in (2.1) [with $r+\lambda$ instead of $r$ ] cannot be optimally stopped at the diagonal $s=m$ in $\langle 0, \infty\rangle \times\langle 0, \infty\rangle$. The latter fact is well-known for diffusions in the maximum process problems of optimal stopping with linear cost (see e.g. Proposition 2.1 in [9]) and only minor modifications are needed to extend the argument to the present case. For this, set $Z_{t}=\sigma B_{t}+\left(r-\sigma^{2} / 2\right) t$ and note 
that the exponential case of (2.1) [ with $r+\lambda$ instead of $r$ ] reduces to the linear case of [9] for the diffusion $Z$ and $c=r+\lambda$ by means of Jensen's inequality as follows:

$$
\begin{aligned}
E\left(e^{-(r+\lambda) \tau} M_{\tau}\right) & =E\left(\exp \left(\max _{0 \leq t \leq \tau} Z_{t}-c \tau\right)\right) \\
& \geq \exp \left(E\left(\max _{0 \leq t \leq \tau} Z_{t}-c \tau\right)\right) .
\end{aligned}
$$

Denoting $\tau_{n}=\inf \left\{t>0 \mid Z_{t} \neq\langle-1 / n, 1 / n\rangle\right\}$ it is easily verified that (cf. Proposition 2.1 in [9]):

$$
E\left(\max _{0 \leq t \leq \tau_{n}} Z_{t}\right) \geq \frac{\delta}{n} \text { and } E\left(\tau_{n}\right) \leq \frac{\kappa}{n^{2}}
$$

for all $n \geq 1$ with some constants $\delta>0$ and $\kappa>0$. Choosing $n$ large enough, upon recalling (3.16), we see that (3.17) shows that it is never optimal to stop at the diagonal in the case of infinite horizon. To derive the same conclusion in the finite horizon case replace $\tau_{n}$ by $\sigma_{n}=\tau_{n} \wedge T$ and note by Markov's inequality and (3.17) that:

$$
\begin{aligned}
E\left(\max _{0 \leq t \leq \tau_{n}} Z_{t}-\max _{0 \leq t \leq \sigma_{n}} Z_{t}\right) & \leq \frac{1}{n} P\left(\tau_{n}>T\right) \\
& \leq \frac{E\left(\tau_{n}\right)}{n T} \leq \frac{\kappa}{n^{3} T}=O\left(n^{-3}\right)
\end{aligned}
$$

which together with (3.16) and (3.17) shows that:

$$
E\left(e^{-(r+\lambda) \sigma_{n}} M_{\sigma_{n}}\right) \geq \exp \left(E\left(\max _{0 \leq t \leq \sigma_{n}} Z_{t}-c \sigma_{n}\right)\right)>1
$$

for $n$ large enough. From (3.19) we see that it is never optimal to stop at the diagonal in the case of finite horizon either, and thus $b$ does not take the value 1 on $[0, T\rangle$ as claimed.

Since the stopping set equals $\bar{D}=\{(t, x) \in[0, T\rangle \times[1, \infty\rangle \mid x \geq b(t)\}$ and $b$ is decreasing, it is easily seen that $b$ is right-continuous on $[0, T\rangle$. Before we pass to the proof of its continuity we first turn to the key principle of optimal stopping in the problem (2.10).

3. We show that the smooth-fit condition (2.13) holds. For this, let $t \in[0, T\rangle$ be given and fixed and set $x=b(t)$. We know that $x>1$ so that there exists $\varepsilon>0$ such that $x-\varepsilon>1$ too. Since $V(t, x)=G(x)$ and $V(t, x-\varepsilon)>G(x-\varepsilon)$, we have:

$$
\frac{V(t, x)-V(t, x-\varepsilon)}{\varepsilon} \leq \frac{G(x)-G(x-\varepsilon)}{\varepsilon}=1
$$

so that by letting $\varepsilon \downarrow 0$ in (3.20) and using that the left-hand derivative $V_{x}^{-}(t, x)$ exists since $y \mapsto V(t, y)$ is convex, we get $V_{x}^{-}(t, x) \leq 1$. To prove the reverse inequality, let $\tau_{\varepsilon}=\tau_{\varepsilon}^{*}(t, x-\varepsilon)$ denote the optimal stopping time for $V(t, x-\varepsilon)$. We then have: 


$$
\begin{aligned}
& \frac{V(t, x)-V(t, x-\varepsilon)}{\varepsilon} \\
\geq & \frac{1}{\varepsilon} \widetilde{E}\left(e^{-\lambda \tau_{\varepsilon}}\left(\frac{\left(x-M_{\tau_{\varepsilon}}\right)^{+}+M_{\tau_{\varepsilon}}}{S_{\tau_{\varepsilon}}}-\frac{\left(x-\varepsilon-M_{\tau_{\varepsilon}}\right)^{+}+M_{\tau_{\varepsilon}}}{S_{\tau_{\varepsilon}}}\right)\right) \\
= & \frac{1}{\varepsilon} \widetilde{E}\left(\frac{e^{-\lambda \tau_{\varepsilon}}}{S_{\tau_{\varepsilon}}}\left(\left(x-M_{\tau_{\varepsilon}}\right)^{+}-\left(x-\varepsilon-M_{\tau_{\varepsilon}}\right)^{+}\right)\right) \\
\geq & \frac{1}{\varepsilon} \widetilde{E}\left(\frac{e^{-\lambda \tau_{\varepsilon}}}{S_{\tau_{\varepsilon}}}\left(\left(x-M_{\tau_{\varepsilon}}\right)^{+}-\left(x-\varepsilon-M_{\tau_{\varepsilon}}\right)^{+}\right) I\left(M_{\tau_{\varepsilon}} \leq x-\varepsilon\right)\right) \\
= & \widetilde{E}\left(\frac{e^{-\lambda \tau_{\varepsilon}}}{S_{\tau_{\varepsilon}}} I\left(M_{\tau_{\varepsilon}} \leq x-\varepsilon\right)\right) \rightarrow 1
\end{aligned}
$$

as $\varepsilon \downarrow 0$ by bounded convergence since $\tau_{\varepsilon} \rightarrow 0$ so that $M_{\tau_{\varepsilon}} \rightarrow 1$ with $1<x-\varepsilon$ and likewise $S_{\tau_{\varepsilon}} \rightarrow 1$. It thus follows from (3.21) that $V_{x}^{-}(t, x) \geq 1$ and therefore $V_{x}^{-}(t, x)=1$. Since $V(t, y)=G(y)$ for $y>x$, it is clear that $V_{x}^{+}(t, x)=1$. We may thus conclude that $y \mapsto V(t, y)$ is $C^{1}$ at $b(t)$ and $V_{x}(t, b(t))=1$ as stated in (2.13).

4. We show that $b$ is continuous on $[0, T]$ and that $b(T-)=1$. For this, note first that since the supremum in (2.10) is attained at the first exit time $\tau_{b}$ from the open set $C$, standard arguments based on the strong Markov property (cf. [3]) imply that $V$ is $C^{1,2}$ on $C$ and satisfies (2.11). Suppose that there exists $t \in\langle 0, T]$ such that $b(t-)>b(t)$ and fix any $x \in[b(t), b(t-)\rangle$. Note that by (2.13) we have:

$$
V(s, x)-x=\int_{x}^{b(s)} \int_{y}^{b(s)} V_{x x}(s, z) d z d y
$$

for each $s \in\langle t-\delta, t\rangle$ where $\delta>0$ with $t-\delta>0$. Since $V_{t}-r x V_{x}+\left(\sigma^{2} / 2\right) x^{2} V_{x x}-$ $\lambda V=0$ in $C$ we see that $\left(\sigma^{2} / 2\right) x^{2} V_{x x}=-V_{t}+r x V_{x}+\lambda V \geq r V_{x}$ in $C$ since $V_{t} \leq 0$ and $V_{x} \geq 0$ upon recalling also that $x \geq 1$ and $\lambda V \geq 0$. Hence we see that there exists $c>0$ such that $V_{x x} \geq c V_{x}$ in $C \cap\{(t, x) \in[0, T\rangle \times[1, \infty\rangle \mid x \leq b(0)\}$, so that this inequality applies in particular to the integrand in (3.22). In this way we get:

$$
V(s, x)-x \geq c \int_{x}^{b(s)} \int_{y}^{b(s)} V_{x}(s, z) d z d y=c \int_{x}^{b(s)}(b(s)-V(s, y)) d y
$$

for all $s \in\langle t-\delta, t\rangle$. Letting $s \uparrow t$ we get:

$$
V(t, x)-x \geq c \int_{x}^{b(t-)}(b(t-)-y) d y=(c / 2)(b(t-)-x)^{2}>0
$$

which is a contradiction since $(t, x)$ belongs to the stopping set $\bar{D}$. This shows that $b$ is continuous on $[0, T]$. Note also that the same argument with $t=T$ shows that $b(T-)=1$.

5. We show that the normal reflection condition (2.14) holds. For this, note first that since $x \mapsto V(t, x)$ is increasing (and convex) on $[1, \infty\rangle$ it follows that $V_{x}(t, 1+) \geq$ 


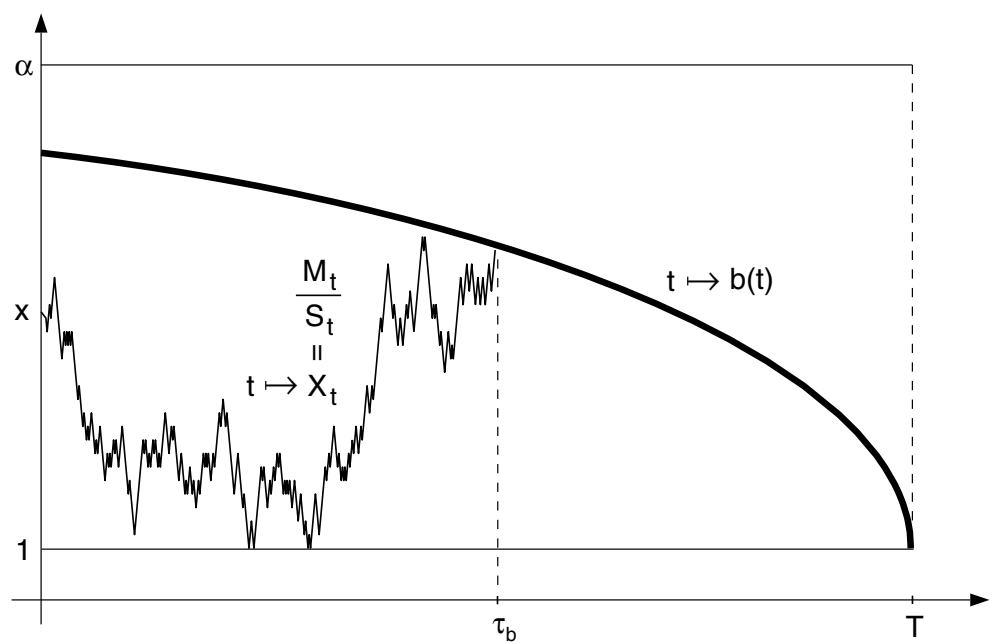

Fig. 1. A computer drawing of the optimal stopping boundary $b$ from Theorem 3.1. The number $\alpha$ is the optimal stopping point in the case of infinite horizon. If the discounting rate $\lambda$ is zero, then $\alpha$ is infinite (i.e. it is never optimal to stop), while $b$ is still finite and looks as above

0 for all $t \in[0, T\rangle$. Suppose that there exists $t \in[0, T\rangle$ such that $V_{x}(t, 1+)>0$. Recalling that $V$ is $C^{1,2}$ on $C$ so that $t \mapsto V_{x}(t, 1+)$ is continuous on $[0, T\rangle$, we see that there exists $\delta>0$ such that $V_{x}(s, 1+) \geq \varepsilon>0$ for all $s \in[t, t+\delta]$ with $t+\delta<T$. Setting $\tau_{\delta}=\tau_{b} \wedge(t+\delta)$ it follows by Itô's formula, using (2.11), and the optional sampling theorem (since $V_{x}$ is bounded) that:

$$
\begin{aligned}
\widetilde{E}_{t, 1}\left(e^{-\lambda \tau_{\delta}}\right. & \left.V\left(t+\tau_{\delta}, X_{t+\tau_{\delta}}\right)\right) \\
& =V(t, 1)+\widetilde{E}_{t, 1}\left(\int_{0}^{\tau_{\delta}} e^{-\lambda u} V_{x}\left(t+u, X_{t+u}\right) d R_{t+u}\right)
\end{aligned}
$$

Since $\left(e^{-\lambda\left(s \wedge \tau_{b}\right)} V\left(t+\left(s \wedge \tau_{b}\right), X_{t+\left(s \wedge \tau_{b}\right)}\right)\right)_{0 \leq s \leq T-t}$ is a martingale under $\widetilde{P}_{t, 1}$, we see that the expression on the left-hand side in (3.25) equals the first term on the right-hand side, and thus:

$$
\widetilde{E}_{t, 1}\left(\int_{0}^{\tau_{\delta}} e^{-\lambda u} V_{x}\left(t+u, X_{t+u}\right) d R_{t+u}\right)=0 .
$$

On the other hand, since $V_{x}\left(t+u, X_{t+u}\right) d R_{t+u}=V_{x}(t+u, 1+) d R_{t+u}$ by (2.7), and $V_{x}(t+u, 1+) \geq \varepsilon>0$ for all $u \in\left[0, \tau_{\delta}\right]$, we see that (3.26) implies that:

$$
\widetilde{E}_{t, 1}\left(\int_{0}^{\tau_{\delta}} d R_{t+u}\right)=0 .
$$

By (2.6) and the optional sampling theorem we see that (3.27) is equivalent to:

$$
\widetilde{E}_{t, 1}\left(X_{t+\tau \delta}\right)-1+r \widetilde{E}_{t, 1}\left(\int_{0}^{\tau_{\delta}} X_{t+u} d u\right)=0
$$


Since $X_{s} \geq 1$ for all $s \in[0, T]$ we see that (3.28) implies that $\tau_{\delta}=0 \widetilde{P}_{t, 1}$-a.s. As clearly this is impossible, we see that $V_{x}(t, 1+)=0$ for all $t \in[0, T\rangle$ as claimed in (2.14).

6. We show that $b$ solves the equation (3.4) on $[0, T]$. For this, set $F(t, x)=$ $e^{-\lambda t} V(t, x)$ and note that $F:[0, T\rangle \times[1, \infty\rangle \rightarrow \mathbb{R}$ is a continuous function satisfying the following conditions:

$$
\begin{aligned}
& F \text { is } C^{1,2} \text { on } C \cup D \\
& F_{t}+\mathbb{L}_{X} F \text { is locally bounded } \\
& x \mapsto F(t, x) \text { is convex } \\
& t \mapsto F_{x}(t, b(t) \pm) \text { is continuous. }
\end{aligned}
$$

To verify these claims, note first that $F(t, x)=e^{-\lambda t} G(x)=e^{-\lambda t} x$ for $(t, x) \in D$ so that the second part of (3.29) is obvious. Similarly, since $F(t, x)=$ $e^{-\lambda t} V(t, x)$ and $V$ is $C^{1,2}$ on $C$, we see that the same is true for $F$, implying the first part of (3.29). For (3.30), note that $\left(F_{t}+\mathbb{L}_{X} F\right)(t, x)=e^{-\lambda t}\left(V_{t}+\mathbb{L}_{X} V-\right.$ $\lambda V)(t, x)=0$ for $(t, x) \in C$ by means of (2.11), and $\left(F_{t}+\mathbb{L}_{X} F\right)(t, x)=$ $e^{-\lambda t}\left(G_{t}+\mathbb{L}_{X} G-\lambda G\right)(t, x)=-(r+\lambda) x e^{-\lambda t}$ for $(t, x) \in D$, implying the claim. [When we say in (3.30) that $F_{t}+\mathbb{L}_{X} F$ is locally bounded, we mean that $F_{t}+\mathbb{L}_{X} F$ is bounded on $K \cap(C \cup D)$ for each compact set $K$ in $[0, T\rangle \times[0, \infty\rangle$.] The condition (3.31) follows by (3.7) above. Finally, recall by (2.13) that $x \mapsto V(t, x)$ is $C^{1}$ at $b(t)$ with $V_{x}(t, b(t))=1$ so that $F_{x}(t, b(t) \pm)=e^{-\lambda t}$ implying (3.32). Let us also note that the condition (3.31) can further be relaxed to the form where $F_{x x}=F_{1}+F_{2}$ on $C \cup D$ where $F_{1}$ is non-negative and $F_{2}$ is continuous on $[0, T\rangle \times[1, \infty\rangle$. This will be referred to below as the relaxed form of (3.29)-(3.32) (for more details see [10]).

Having a continuous function $F:[0, T\rangle \times[1, \infty\rangle \rightarrow \mathbb{R}$ satisfying (3.29)-(3.32) one finds in exactly the same way as (2.30) in [10] is derived from (2.26) in [10] that for $t \in[0, T\rangle$ the following change-of-variable formula holds:

$$
\begin{aligned}
F\left(t, X_{t}\right)= & F\left(0, X_{0}\right)+\int_{0}^{t}\left(F_{t}+\mathbb{L}_{X} F\right)\left(s, X_{s}\right) I\left(X_{s} \neq b(s)\right) d s \\
& +\int_{0}^{t} F_{x}\left(s, X_{s}\right) \sigma X_{s} I\left(X_{s} \neq b(s)\right) d \widehat{B}_{s} \\
& +\int_{0}^{t} F_{x}\left(s, X_{s}\right) I\left(X_{s} \neq b(s)\right) d R_{s} \\
& +\frac{1}{2} \int_{0}^{t}\left(F_{x}\left(s, X_{s}+\right)-F_{x}\left(s, X_{s}-\right)\right) I\left(X_{s}=b(s)\right) d \ell_{s}^{b}(X)
\end{aligned}
$$

where $\ell_{s}^{b}(X)$ is the local time of $X$ at the curve $b$ given by:

$$
\ell_{s}^{b}(X)=\mathbb{P}-\lim _{\varepsilon \downarrow 0} \frac{1}{2 \varepsilon} \int_{0}^{s} I\left(b(r)-\varepsilon<X_{r}<b(r)+\varepsilon\right) \sigma^{2} X_{r}^{2} d r
$$

and $d \ell_{s}^{b}(X)$ refers to the integration with respect to the continuous increasing function $s \mapsto \ell_{s}^{b}(X)$. Note also that the formula (3.33) remains valid if $b$ is replaced 
by any other continuous function of bounded variation $c:[0, T] \rightarrow \mathbb{R}$ for which (3.29)-(3.32) hold with $C$ and $D$ defined in the same way.

Applying (3.33) to $e^{-\lambda s} V\left(t+s, X_{t+s}\right)$ under $\widetilde{P}_{t, x}$ with $(t, x) \in[0, T\rangle \times[1, \infty\rangle$ yields:

$$
\begin{aligned}
& e^{-\lambda s} V\left(t+s, X_{t+s}\right) \\
& =V(t, x)+\int_{0}^{s} e^{-\lambda u}\left(V_{t}+\mathbb{L}_{X} V-\lambda V\right)\left(t+u, X_{t+u}\right) d u+M_{s} \\
& =V(t, x)+\int_{0}^{s} e^{-\lambda u}\left(G_{t}+\mathbb{L}_{X} G-\lambda G\right)\left(t+u, X_{t+u}\right) I\left(X_{t+u} \geq b(t+u)\right) d u+M_{s} \\
& =V(t, x)-(r+\lambda) \int_{0}^{s} e^{-\lambda u} X_{t+u} I\left(X_{t+u} \geq b(t+u)\right) d u+M_{s}
\end{aligned}
$$

upon using (2.11), (2.12)+(2.16), (2.14), (2.13) and $G_{t}+\mathbb{L}_{X} G-\lambda G=-(r+\lambda) G$, where we set $M_{s}=\int_{0}^{s} e^{-\lambda u} V_{x}\left(t+u, X_{t+u}\right) \sigma X_{t+u} d \widehat{B}_{t+u}$ for $0 \leq s \leq T-t$. Since $0 \leq V_{x} \leq 1$ on $[0, T] \times[1, \infty\rangle$, it is easily verified that $\left(M_{s}\right)_{0 \leq s \leq T-t}$ is a martingale, so that $\widetilde{E}_{t, x}\left(M_{s}\right)=0$ for all $0 \leq s \leq T-t$. Inserting $s=T-t$ in (3.35), using that $V(T, x)=G(x)=x$ for all $x \in[1, \infty\rangle$, and taking the $\widetilde{P}_{t, x}$-expectation in the resulting identity, we get:

$$
\begin{aligned}
& e^{-\lambda(T-t)} \widetilde{E}_{t, x}\left(X_{T}\right) \\
& =V(t, x)-(r+\lambda) \int_{0}^{T-t} e^{-\lambda u} \widetilde{E}_{t, x}\left(X_{t+u} I\left(X_{t+u} \geq b(t+u)\right)\right) d u
\end{aligned}
$$

for all $(t, x) \in[0, T\rangle \times[1, \infty\rangle$. By (3.1) and (3.2) we see that (3.36) is the early exercise premium representation (3.5). Recalling that $V(t, x)=G(x)=x$ for $x \geq b(t)$, and setting $x=b(t)$ in (3.36), we see that $b$ satisfies the equation (3.4) as claimed.

7.1. We show that $b$ is the unique solution of the equation (3.4) in the class of continuous decreasing functions $c:[0, T] \rightarrow \mathbb{R}$ satisfying $c(t)>1$ for all $0 \leq t<$ $T$. The proof of this fact will be carried out in several remaining subsections to the end of the main proof. Let us thus assume that a function $c$ belonging to the class described above solves (3.4), and let us show that this $c$ must then coincide with the optimal stopping boundary $b$.

For this, in view of (3.36), let us introduce the function:

$$
\begin{aligned}
U^{c}(t, x)= & e^{-\lambda(T-t)} \widetilde{E}_{t, x}\left(X_{T}\right) \\
& +(r+\lambda) \int_{0}^{T-t} e^{-\lambda u} \widetilde{E}_{t, x}\left(X_{t+u} I\left(X_{t+u} \geq c(t+u)\right)\right) d u
\end{aligned}
$$

for $(t, x) \in[0, T\rangle \times[1, \infty\rangle$. Using (3.1) and (3.2) as in (3.5) we see that (3.37) reads:

$$
U^{c}(t, x)=e^{-\lambda(T-t)} F(T-t, x)+(r+\lambda) \int_{0}^{T-t} e^{-\lambda u} G(u, x, c(t+u)) d u
$$


for $(t, x) \in[0, T\rangle \times[1, \infty\rangle$. A direct inspection of the expressions in (3.38) using (3.1)-(3.3) shows that $U_{x}^{c}$ is continuous on $[0, T\rangle \times[1, \infty\rangle$.

7.2. In accordance with (3.5) define a function $V^{c}:[0, T\rangle \times[1, \infty\rangle \rightarrow \mathbb{R}$ by setting $V^{c}(t, x)=U^{c}(t, x)$ for $x<c(t)$ and $V^{c}(t, x)=G(x)$ for $x \geq c(t)$ when $0 \leq t<T$. Note that since $c$ solves (3.4) we have that $V^{c}$ is continuous on $[0, T\rangle \times[1, \infty\rangle$, i.e. $V^{c}(t, x)=U^{c}(t, x)=G(x)$ for $x=c(t)$ when $0 \leq t<T$. Let $C$ and $D$ be defined by means of $c$ as in (2.17) and (2.18) respectively.

Standard arguments based on the Markov property (or a direct verification) show that $V^{c}$ i.e. $U^{c}$ is $C^{1,2}$ on $C$ and that:

$$
\begin{aligned}
& V_{t}^{c}+\mathbb{L}_{X} V^{c}=\lambda V^{c} \text { in } C \\
& V_{x}^{c}(t, 1+)=0
\end{aligned}
$$

for all $t \in[0, T\rangle$. Moreover, since $U_{x}^{c}$ is continuous on $[0, T\rangle \times[1, \infty\rangle$ we see that $V_{x}^{c}$ is continuous on $\bar{C}$. Finally, it is obvious that $V^{c}$ i.e. $G$ is $C^{1,2}$ on $\bar{D}$.

7.3. Summarizing the preceding conclusions one can easily verify that the function $F:[0, T\rangle \times[1, \infty\rangle \rightarrow \mathbb{R}$ defined by $F(t, x)=e^{-\lambda t} V^{c}(t, x)$ satisfies (3.29)(3.32) (in the relaxed form) so that (3.33) can be applied. In this way, under $P_{t, x}$ with $(t, x) \in[0, T\rangle \times[1, \infty\rangle$ given and fixed, using (3.40) we get:

$$
\begin{aligned}
& e^{-\lambda s} V^{c}\left(t+s, X_{t+s}\right)=V^{c}(t, x) \\
& \quad+\int_{0}^{s} e^{-\lambda u}\left(V_{t}^{c}+\mathbb{L}_{X} V^{c}-\lambda V^{c}\right)\left(t+u, X_{t+u}\right) I\left(X_{t+u} \neq c(t+u)\right) d u+M_{s}^{c} \\
& \quad+\frac{1}{2} \int_{0}^{s} e^{-\lambda u} \Delta_{x} V_{x}^{c}(t+u, c(t+u)) d \ell_{u}^{c}(X)
\end{aligned}
$$

where $M_{s}^{c}=\int_{0}^{s} e^{-\lambda u} V_{x}^{c}\left(t+u, X_{t+u}\right) \sigma X_{t+u} I\left(X_{t+u} \neq c(t+u)\right) d \widehat{B}_{t+u}$ and we set $\Delta_{x} V_{x}^{c}(v, c(v))=V_{x}^{c}(v, c(v)+)-V_{x}^{c}(v, c(v)-)$ for $t \leq v \leq T$. Moreover, it is readily seen from the explicit expression for $V_{x}^{c}$ obtained using (3.38) above that $\left(M_{s}^{c}\right)_{0 \leq s \leq T-t}$ is a martingale under $\widetilde{P}_{t, x}$ so that $\widetilde{E}_{t, x}\left(M_{s}^{c}\right)=0$ for each $0 \leq s \leq T-t$.

7.4. Setting $s=T-t$ in (3.41) and then taking the $\widetilde{P}_{t, x}$-expectation, using that $V^{c}(T, x)=G(x)$ for all $x \geq 1$ and that $V^{c}$ satisfies (3.39) in $C$, we get:

$$
\begin{aligned}
& e^{-\lambda(T-t)} \widetilde{E}_{t, x}\left(X_{T}\right)=V^{c}(t, x) \\
& \quad-(r+\lambda) \int_{0}^{T-t} e^{-\lambda u} \widetilde{E}_{t, x}\left(X_{t+u} I\left(X_{t+u} \geq c(t+u)\right)\right) d u \\
& \quad+\frac{1}{2} \int_{0}^{T-t} e^{-\lambda u} \Delta_{x} V_{x}^{c}(t+u, c(t+u)) d_{u} \widetilde{E}_{t, x}\left(\ell_{u}^{c}(X)\right)
\end{aligned}
$$

for all $(t, x) \in[0, T\rangle \times[1, \infty\rangle$. Comparing (3.42) with (3.37), and recalling the definition of $V^{c}$ in terms of $U^{c}$ and $G$, we get: 


$$
\begin{aligned}
& \int_{0}^{T-t} e^{-\lambda u} \Delta_{x} V_{x}^{c}(t+u, c(t+u)) d_{u} \widetilde{E}_{t, x}\left(\ell_{u}^{c}(X)\right) \\
& =2\left(U^{c}(t, x)-G(x)\right) I(x \geq c(t))
\end{aligned}
$$

for all $0 \leq t<T$ and $x \geq 1$, where $I(x \geq c(t))$ equals 1 if $x \geq c(t)$ and 0 if $x<c(t)$.

7.5. From (3.43) we see that if we are to prove that:

$$
x \mapsto V^{c}(t, x) \text { is } C^{1} \text { at } c(t)
$$

for each $0 \leq t<T$ given and fixed, then it will follow that:

$$
U^{c}(t, x)=G(x) \text { for all } x \geq c(t) .
$$

On the other hand, if we know that (3.45) holds, then using the general fact:

$$
\begin{aligned}
& \left.\frac{\partial}{\partial x}\left(U^{c}(t, x)-G(x)\right)\right|_{x=c(t)} \\
& =V_{x}^{c}(t, c(t)-)-V_{x}^{c}(t, c(t)+)=-\Delta_{x} V_{x}^{c}(t, c(t))
\end{aligned}
$$

for all $0 \leq t<T$, we see that (3.44) holds too (since $U_{x}^{c}$ is continuous). The equivalence of (3.44) and (3.45) suggests that instead of dealing with the equation (3.43) in order to derive (3.44) above we may rather concentrate on establishing (3.45) directly.

7.6. To derive (3.45) first note that standard arguments based on the Markov property (or a direct verification) show that $U^{c}$ is $C^{1,2}$ on $D$ and that:

$$
U_{t}^{c}+\mathbb{L}_{X} U^{c}-\lambda U^{c}=-(r+\lambda) G \text { in } D .
$$

Since the function $F:[0, T\rangle \times[1, \infty\rangle \rightarrow \mathbb{R}$ defined by $F(t, x)=e^{-\lambda t} U^{c}(t, x)$ is continuous and satisfies (3.29)-(3.32) (in the relaxed form), we see that (3.33) can be applied just like in (3.41) with $U^{c}$ instead of $V^{c}$, and this yields:

$$
\begin{aligned}
& e^{-\lambda s} U^{c}\left(t+s, X_{t+s}\right)=U^{c}(t, x) \\
& \quad-(r+\lambda) \int_{0}^{s} e^{-\lambda u} X_{t+u} I\left(X_{t+u} \geq c(t+u)\right) d u+\widetilde{M}_{s}^{c}
\end{aligned}
$$

upon using (3.39)+(3.40) and (3.47) as well as that $\Delta_{x} U_{x}^{c}(t+u, c(t+u))=0$ for $0 \leq u \leq s$ since $U_{x}^{c}$ is continuous. In (3.48) we have $\widetilde{M}_{s}^{c}=\int_{0}^{s} e^{-\lambda u} U_{x}^{c}(t+$ $\left.u, X_{t+u}\right) \sigma X_{t+u} I\left(X_{t+u} \neq c(t+u)\right) d \widehat{B}_{t+u}$ and $\left(\widetilde{M}_{s}^{c}\right)_{0 \leq s \leq T-t}$ is a martingale under $P_{t, x}$.

Next note that Itô's formula implies:

$$
e^{-\lambda s} G\left(X_{t+s}\right)=G(x)-(r+\lambda) \int_{0}^{s} e^{-\lambda u} X_{t+u} d u+M_{s}+\int_{0}^{s} e^{-\lambda u} d R_{t+u}
$$


upon using that $G_{t}+\mathbb{L}_{X} G-r G=-(r+\lambda) G$ as well as that $G_{x}\left(t+u, X_{t+u}\right)=1$ for $0 \leq u \leq s$. In (3.49) we have $M_{s}=\int_{0}^{s} e^{-\lambda u} \sigma X_{t+u} d \widehat{B}_{t+u}$ and $\left(M_{s}\right)_{0 \leq s \leq T-t}$ is a martingale under $P_{t, x}$.

For $x \geq c(t)$ consider the stopping time:

$$
\sigma_{c}=\inf \left\{0 \leq s \leq T-t \mid X_{t+s} \leq c(t+s)\right\} .
$$

Then using that $U^{c}(t, c(t))=G(c(t))$ for all $0 \leq t<T$ since $c$ solves (3.4), and that $U^{c}(T, x)=G(x)$ for all $x \geq 1$ by (3.37), we see that $U^{c}\left(t+\sigma_{c}, X_{t+\sigma_{c}}\right)=$ $G\left(X_{t+\sigma_{c}}\right)$. Hence from (3.48) and (3.49) using the optional sampling theorem we find:

$$
\begin{aligned}
U^{c}(t, x)= & E_{t, x}\left(e^{-\lambda \sigma_{c}} U^{c}\left(t+\sigma_{c}, X_{t+\sigma_{c}}\right)\right) \\
& +(r+\lambda) E_{t, x}\left(\int_{0}^{\sigma_{c}} e^{-\lambda u} X_{t+u} I\left(X_{t+u} \geq c(t+u)\right) d u\right) \\
= & E_{t, x}\left(e^{-r \sigma_{c}} G\left(X_{t+\sigma_{c}}\right)\right) \\
& +(r+\lambda) E_{t, x}\left(\int_{0}^{\sigma_{c}} e^{-\lambda u} X_{t+u} I\left(X_{t+u} \geq c(t+u)\right) d u\right) \\
= & G(x)-(r+\lambda) E_{t, x}\left(\int_{0}^{\sigma_{c}} e^{-\lambda u} X_{t+u} d u\right) \\
& +(r+\lambda) E_{t, x}\left(\int_{0}^{\sigma_{c}} e^{-\lambda u} X_{t+u} I\left(X_{t+u} \geq c(t+u)\right) d u\right)=G(x)
\end{aligned}
$$

since $X_{t+u} \geq c(t+u)>1$ for all $0 \leq u \leq \sigma_{c}$. This establishes (3.45) and thus (3.44) holds too.

It may be noted that a shorter but somewhat less revealing proof of (3.45) [and (3.44)] can be obtained by verifying directly (using the Markov property only) that the process:

$$
e^{-\lambda s} U^{c}\left(t+s, X_{t+s}\right)+(r+\lambda) \int_{0}^{s} e^{-\lambda u} X_{t+u} I\left(X_{t+u} \geq c(t+u)\right) d u
$$

is a martingale under $P_{t, x}$ for $0 \leq s \leq T-t$. This verification moreover shows that the martingale property of (3.52) does not require that $c$ is increasing but only measurable. Taken together with the rest of the proof below this shows that the claim of uniqueness for the equation (3.4) holds in the class of continuous functions $c:[0, T] \rightarrow \mathbb{R}$ such that $c(t)>1$ for all $0<t<T$.

7.7. Consider the stopping time:

$$
\tau_{c}=\inf \left\{0 \leq s \leq T-t \mid X_{t+s} \geq c(t+s)\right\} .
$$

Note that (3.41) using (3.39) and (3.44) reads:

$$
\begin{aligned}
& e^{-\lambda s} V^{c}\left(t+s, X_{t+s}\right)=V^{c}(t, x) \\
& \quad-(r+\lambda) \int_{0}^{s} e^{-\lambda u} X_{t+u} I\left(X_{t+u} \geq c(t+u)\right) d u+M_{s}^{c}
\end{aligned}
$$


where $\left(M_{s}^{c}\right)_{0 \leq s \leq T-t}$ is a martingale under $\widetilde{P}_{t, x}$. Thus $\widetilde{E}_{t, x}\left(M_{\tau_{c}}^{c}\right)=0$, so that after inserting $\tau_{c}$ in place of $s$ in (3.54), it follows upon taking the $\widetilde{P}_{t, x}$-expectation that:

$$
V^{c}(t, x)=\widetilde{E}_{t, x}\left(e^{-\lambda \tau_{c}} X_{t+\tau_{c}}\right)
$$

for all $(t, x) \in[0, T\rangle \times[1, \infty\rangle$ where we use that $V^{c}(t, x)=G(x)=x$ for $x \geq c(t)$ or $t=T$. Comparing (3.55) with (2.10) we see that:

$$
V^{c}(t, x) \leq V(t, x)
$$

for all $(t, x) \in[0, T\rangle \times[1, \infty\rangle$.

7.8. Let us now show that $b \geq c$ on $[0, T]$. For this, recall that by the same arguments as for $V^{c}$ we also have:

$$
\begin{aligned}
& e^{-\lambda s} V\left(t+s, X_{t+s}\right)=V(t, x) \\
& \quad-(r+\lambda) \int_{0}^{s} e^{-\lambda u} X_{t+u} I\left(X_{t+u} \geq b(t+u)\right) d u+M_{s}^{b}
\end{aligned}
$$

where $\left(M_{s}^{b}\right)_{0 \leq s \leq T-t}$ is a martingale under $\widetilde{P}_{t, x}$. Fix $(t, x) \in[0, T\rangle \times[1, \infty\rangle$ such that $x>b(t) \vee c(t)$ and consider the stopping time:

$$
\sigma_{b}=\inf \left\{0 \leq s \leq T-t \mid X_{t+s} \leq b(t+s)\right\} .
$$

Inserting $\sigma_{b}$ in place of $s$ in (3.54) and (3.57) and taking the $\widetilde{P}_{t, x}$-expectation, we get:

$$
\begin{aligned}
& \widetilde{E}_{t, x}\left(e^{-\lambda \sigma_{b}} V^{c}\left(t+\sigma_{b}, X_{t+\sigma_{b}}\right)\right) \\
& =x-(r+\lambda) \widetilde{E}_{t, x}\left(\int_{0}^{\sigma_{b}} e^{-\lambda u} X_{t+u} I\left(X_{t+u} \geq c(t+u)\right) d u\right) \\
& \widetilde{E}_{t, x}\left(e^{-\lambda \sigma_{b}} V\left(t+\sigma_{b}, X_{t+\sigma_{b}}\right)\right) \\
& =x-(r+\lambda) \widetilde{E}_{t, x}\left(\int_{0}^{\sigma_{b}} e^{-\lambda u} X_{t+u} d u\right) .
\end{aligned}
$$

Hence by (3.56) we see that:

$$
\widetilde{E}_{t, x}\left(\int_{0}^{\sigma_{b}} e^{-\lambda u} X_{t+u} I\left(X_{t+u} \geq c(t+u)\right) d u\right) \geq \widetilde{E}_{t, x}\left(\int_{0}^{\sigma_{b}} e^{-\lambda u} X_{t+u} d u\right)
$$

from where it follows by the continuity of $c$ and $b$, using $X_{t+u}>0$, that $b(t) \geq c(t)$ for all $t \in[0, T]$.

7.9. Finally, let us show that $c$ must be equal to $b$. For this, assume that there is $t \in\langle 0, T\rangle$ such that $b(t)>c(t)$, and pick $x \in\langle c(t), b(t)\rangle$. Under $\widetilde{P}_{t, x}$ consider the stopping time $\tau_{b}$ from (2.19). Inserting $\tau_{b}$ in place of $s$ in (3.54) and (3.57) and taking the $\widetilde{P}_{t, x}$-expectation, we get: 


$$
\begin{aligned}
& \widetilde{E}_{t, x}\left(e^{-\lambda \tau_{b}} X_{t+\tau_{b}}\right)=V^{c}(t, x) \\
& \quad-(r+\lambda) \widetilde{E}_{t, x}\left(\int_{0}^{\tau_{b}} e^{-\lambda u} X_{t+u} I\left(X_{t+u} \geq c(t+u)\right) d u\right) \\
& \widetilde{E}_{t, x}\left(e^{-\lambda \tau_{b}} X_{t+\tau_{b}}\right)=V(t, x) .
\end{aligned}
$$

Hence by (3.56) we see that:

$$
\widetilde{E}_{t, x}\left(\int_{0}^{\tau_{b}} e^{-\lambda u} X_{t+u} I\left(X_{t+u} \geq c(t+u)\right) d u\right) \leq 0
$$

from where it follows by the continuity of $c$ and $b$ using $X_{t+u}>0$ that such a point $x$ cannot exist. Thus $c$ must be equal to $b$, and the proof is complete.

Note added in proof. I thank Andreas Kyprianou for stimulating discussions and the preprint [2]. I also thank Erik Ekström for his interest in the first draft of the present paper and for sending me his preprint [4]. Both [2] and [4] provide useful additions to the main result of the present paper.

\section{References}

1. Carr, P., Jarrow, R., Myneni, R.: Alternative characterizations of American put options. Math. Finance 2, 78-106 (1992)

2. Duistermaat, J.J., Kyprianou, A.E., van Schaik, K.: Finite expiry Russian options. Preprint, University of Utrecht 2003

3. Dynkin, E.B.: Markov processes. Berlin Heidelberg New York: Springer 1965

4. Ekström, E.: Russian options with a finite time horizon. Preprint, University of Uppsala 2003

5. Jacka, S.D.: Optimal stopping and the American put. Math. Finance 1, 1-14 (1991)

6. Karatzas, I., Shreve S.E.: Methods of mathematical finance. Berlin Heidelberg New York: Springer 1998

7. Kim, I.J.: The analytic valuation of American options. Rev. Financial Stud. 3, 547-572 (1990)

8. Kolodner, I.I.: Free boundary problem for the heat equation with applications to problems of change of phase I. General method of solution. Comm. Pure Appl. Math. 9, 1-31 (1956)

9. Peskir, G.: Optimal stopping of the maximum process: The maximality principle. Ann. Probab. 26, 1614-1640 (1998)

10. Peskir, G.: On the American option problem. Research Report No. 431, Department of Theoretical Statistics, Aarhus (13 pp). Math. Finance (forthcoming)

11. Shepp, L.A., Shiryaev, A.N.: The Russian option: Reduced regret. Ann. Appl. Probab. 3, 631-640 (1993)

12. Shepp, L.A., Shiryaev, A.N.: A new look at the Russian option. Theory Probab. Appl. 39, 103-119 (1994)

13. Shiryaev, A.N.: Optimal stopping rules. Berlin Heidelberg New York: Springer 1978

14. Shiryaev, A.N.: Essentials of stochastic finance (facts, models, theory). World Scientific 1999 